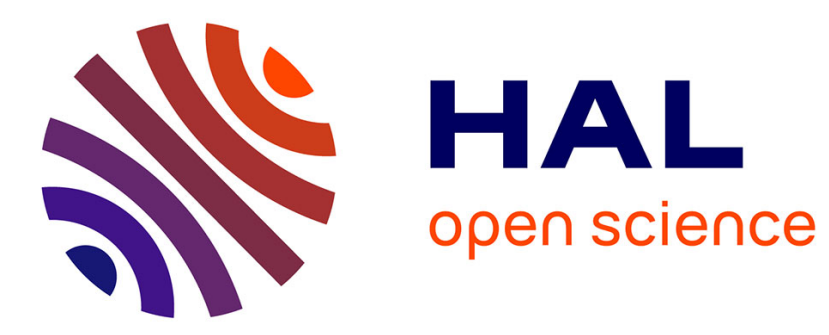

\title{
Ge-rich SiGe waveguides for mid-infrared photonics
}

Joan Manel Ramírez, Vladyslav Vakarin, Papichaya Chaisakul, Jacopo

Frigerio, Andrea Ballabio, Clement Gilles, Daniel Chrastina, Qiankun Liu, Gregory Maisons, Xavier Le Roux, et al.

\section{- To cite this version:}

Joan Manel Ramírez, Vladyslav Vakarin, Papichaya Chaisakul, Jacopo Frigerio, Andrea Ballabio, et al.. Ge-rich SiGe waveguides for mid-infrared photonics. SPIE Photonics West, Jan 2017, San Francisco, CA, United States. 10.1117/12.2252832 . hal-01616379

\section{HAL Id: hal-01616379 \\ https://hal.science/hal-01616379}

Submitted on 13 Oct 2017

HAL is a multi-disciplinary open access archive for the deposit and dissemination of scientific research documents, whether they are published or not. The documents may come from teaching and research institutions in France or abroad, or from public or private research centers.
L'archive ouverte pluridisciplinaire HAL, est destinée au dépôt et à la diffusion de documents scientifiques de niveau recherche, publiés ou non, émanant des établissements d'enseignement et de recherche français ou étrangers, des laboratoires publics ou privés. 


\title{
Ge-rich SiGe waveguides for mid-infrared photonics
}

\author{
Joan Manel Ramírez, ${ }^{1}$ Vladyslav Vakarin, ${ }^{1}$ Papichaya Chaisakul, ${ }^{1}$ Jacopo Frigerio, ${ }^{2}$, Andrea \\ Ballabio, ${ }^{2}$ Clément Gilles, ${ }^{3}$ Daniel Chrastina, ${ }^{2}$ Qiankun Liu, ${ }^{1}$ Grégory Maisons, ${ }^{3}$ Xavier Le Roux, ${ }^{1}$ \\ Laurent Vivien, ${ }^{1}$ Mathieu Carras, ${ }^{3}$ Giovanni Isella, ${ }^{2}$ Delphine Marris-Morini ${ }^{1}$ \\ ${ }^{1}$ Centre de Nanosciences et Nanotechnologies, Univ. Paris-Sud, CNRS UMR 9100, \\ Université Paris Saclay, Bâtiment 220, 91405 Orsay Cedex, France; \\ ${ }^{2}$ L-NESS, Dipartimento di Fisica, Politecnico di Milano, Polo di Como, Via Anzani 42, I 22100 \\ Como, Italy \\ ${ }^{3}$ MirSense, 86 Rue de Paris, 91400 Orsay, France
}

\begin{abstract}
The extension of silicon photonics towards the mid infrared (mid-IR) spectral range has recently attracted a lot of attention. The development of photonic devices operating at these wavelengths is crucial for many applications including environmental and chemical sensing, astronomy and medicine. Recent works regarding the development of Ge-rich SiGe waveguides on graded buffer layers will be presented. It will be shown that these waveguides demonstrate low loss and strong mode confinement for a large range of wavelengths and that they have a good potential for being a major building block of mid-infrared photonic integrated circuits.
\end{abstract}

Keywords: Silicon photonics, mid-IR photonics, Germanium, waveguide, non-linear optics

\section{INTRODUCTION}

The demonstration of integrated photonic circuits in the mid-IR wavelength range $(2-20 \mu \mathrm{m})$ is a major issue for the realization of sensors for many applications requiring portable spectroscopic detection schemes. In this context silicon photonics integrated circuits present strong interest to offer compact, low cost, lightweight and low power consumption systems. Among the different materials available in silicon photonics, Germanium $(\mathrm{Ge})$ and Silicon-Germanium (SiGe) alloys with a strong Ge concentration are particularly interesting because of the wide transparency window of Ge from 1 to $15 \mu \mathrm{m}$ [1]. A large variety of passive structures based on Ge strip waveguides on a Si substrate have been demonstrated in the mid-IR range, such as low-loss waveguides, wavelength (de)multiplexer, grating couplers or MMI [2-5]. Graded $\mathrm{SiGe} / \mathrm{Si}$ waveguides have also been investigated by ramping the Ge concentration in the $\mathrm{Si}_{1-\mathrm{x}} \mathrm{Ge}_{\mathrm{x}}$ core up to $\approx 40 \%[6]$.

In parallel Ge-rich SiGe waveguides on graded SiGe substrates were used in the near-IR wavelength range, to integrate active devices (modulator and photodetector) based on Ge-rich active region on Si substrate [7]. Interestingly such new photonic platform presents also strong advantages for the development of mid-IR photonics integrated circuits, thanks to the large transparency window of Ge-rich $\mathrm{Si}_{1-\mathrm{x}} \mathrm{Ge}_{\mathrm{x}}$ in the mid-IR range, which allows an extension of the maximum operating wavelength beyond $8 \mu \mathrm{m}$ (absorption of the silicon). Indeed the use of a graded buffer can be used to confine the light in the Ge-rich region, far away from the Si-rich area in the waveguide core, which reduces the optical loss of the guided mode. The first consequence is the possibility to extend the wavelength range beyond Si absorption limit. As a second advantage, germanium has a much higher third order nonlinearity coefficient than silicon, with a $\chi^{3}$ coefficient predicted to be up to 10 times higher [8]. The implementation of Ge-rich material using graded SiGe waveguides provides thus good prospects to develop nonlinear mid-IR optical devices, such as broadband optical sources.

As a first step towards the development of such new photonic integrated circuit platform for mid-IR operation passive waveguides have been designed, fabricated and characterized, to demonstrate the possibility to guide light with low 
optical losses. Then waveguides have been designed to optimize nonlinear operation, i.e. to ensure simultaneously small nonlinear effective area as well as flat and small anomalous dispersion.

\section{EXPERIMENTAL DEMONSTRATION OF LOW LOSS OPTICAL WAVEGUIDE IN THE MID IR}

\subsection{Ge-rich SiGe waveguide design and fabrication.}

The Ge-rich SiGe waveguides are based on the epilayer reported in Fig 1(a). The 2- $\mu$ m thick Si0.2Ge0.8 waveguide core is grown on a $11 \mu \mathrm{m}$ graded SiGe buffer from Si to Si0.21Ge0.79, using Low Energy Plasma Enhanced Chemical Vapor Deposition (LEPECVD). This approach allows an efficient reduction of the number of threading dislocations (TDD) to $3 \times 10^{6} \mathrm{~cm}^{-2}$ by gradual accommodation of the Si-Ge lattice mismatch in the layer stack [9]. In addition the linear refractive index increase (as shown in fig. 1(b) due to the gradual increase of the Ge content in SiGe alloy allows the confinement of light at the top of the $\mathrm{Si}_{0.2} \mathrm{Ge}_{0.8}$ layer. Rib waveguides of different widths were defined by etching the Ge-rich $\mathrm{Si}_{0.2} \mathrm{Ge}_{0.8}$ layer down to $1.5 \mu \mathrm{m}$ using Inductively Coupled Plasma (ICP) technique (Fig 1.c). The good confinement of light at $4.6 \mu \mathrm{m}$ is illustrated by the optical mode calculation for two waveguides widths $(3.5$ and $8.5 \mu \mathrm{m})$ (Fig 1.d).

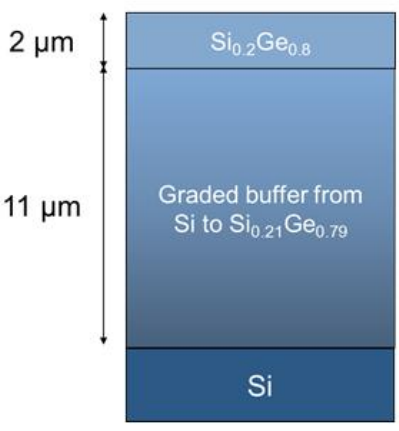

(a)

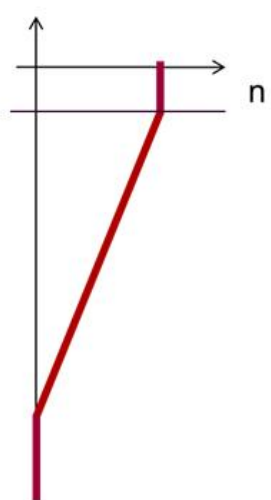

(b)

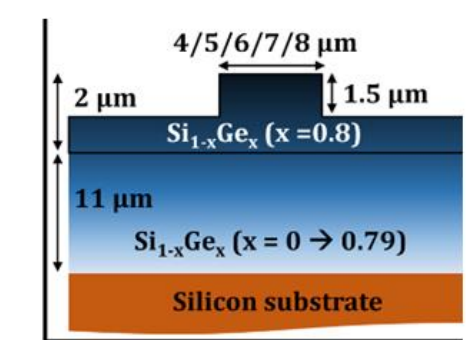

(c)

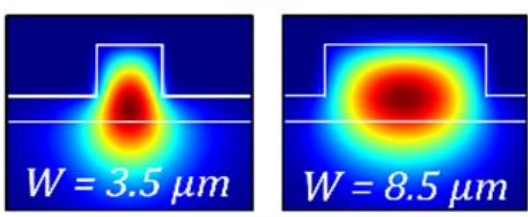

(d) $\quad \lambda=4.6 \mu \mathrm{m}$

Figure 1. Cut-view of the Ge-rich SiGe waveguides (a): epilayer grown by LEPECVD in standard Si substrate. (b) : refractive index profile along the growth direction (c) : schematic view of the fabricated rib waveguides (d) : optical mode calculation at the wavelength of $4.6 \mu \mathrm{m}$ illustrating the good confinement of light.

\subsection{Characterization}

A cut-back technique was used to characterize optical loss of the waveguides. The sample design is seen in the SEM picture of Fig. 2a, showing the deep-etch step to obtain the different straight waveguide lengths from 3 to $6 \mathrm{~mm}$ long. Waveguide characterization was performed using the set-up reported in Fig 2.b. Light at $4.6 \mu \mathrm{m}$ from a quantum cascade laser (QCL) laser is coupled to a mid-IR single mode micro-structured optical fiber for waveguide butt-coupling. A freespace mid-IR collection system using a plano-convex lens is used to collect the output light, which goes through a polarizer and is sent to a mid-IR camera.

As an example of measurement, the transmission of $7 \mu \mathrm{m}$-width waveguide is reported in Fig 3, as a function of waveguide length from 3 to $6 \mathrm{~mm}$. Propagation losses of $1.5 \mathrm{~dB} / \mathrm{cm}$ (resp. $2 \mathrm{~dB} / \mathrm{cm}$ ) can be deduced for TE polarization (resp. TM polarization). Similar losses have been obtained for waveguide widths from 4 to $8 \mu$ m. Coupling losses have 
also been investigated. Low coupling losses around $5 \mathrm{~dB} /$ facet are obtained for waveguide widths of $7 \mu \mathrm{m}$ and larger. They increase smoothly up to 6-7 dB when the width is reduced down to $4 \mu \mathrm{m}$. This result indicates that when waveguide width lower than $7 \mu \mathrm{m}$ are required, the light coupling could be improved using a taper with larger width at the facet of the waveguide.

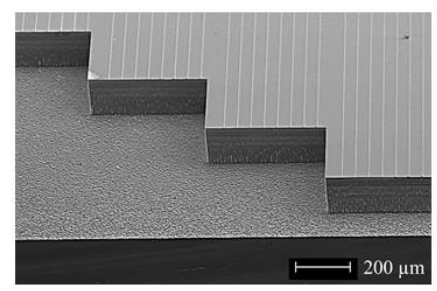

(a)

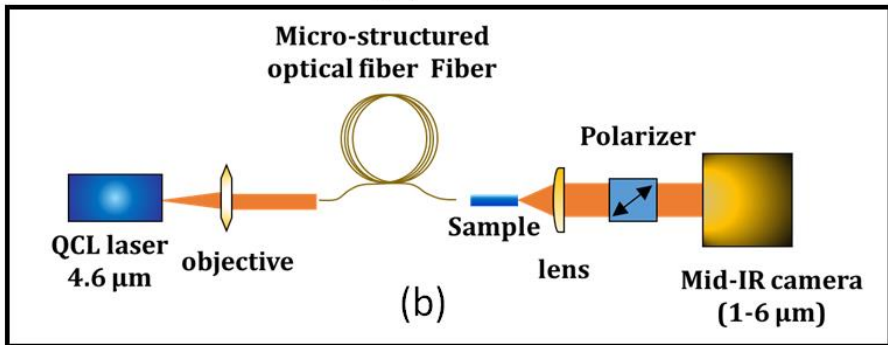

Figure 2. (a) SEM view of the fabricated devices, showing straight waveguides with different lengths for cut-back technique. (b)Experimental set-up used to characterize the optical losses

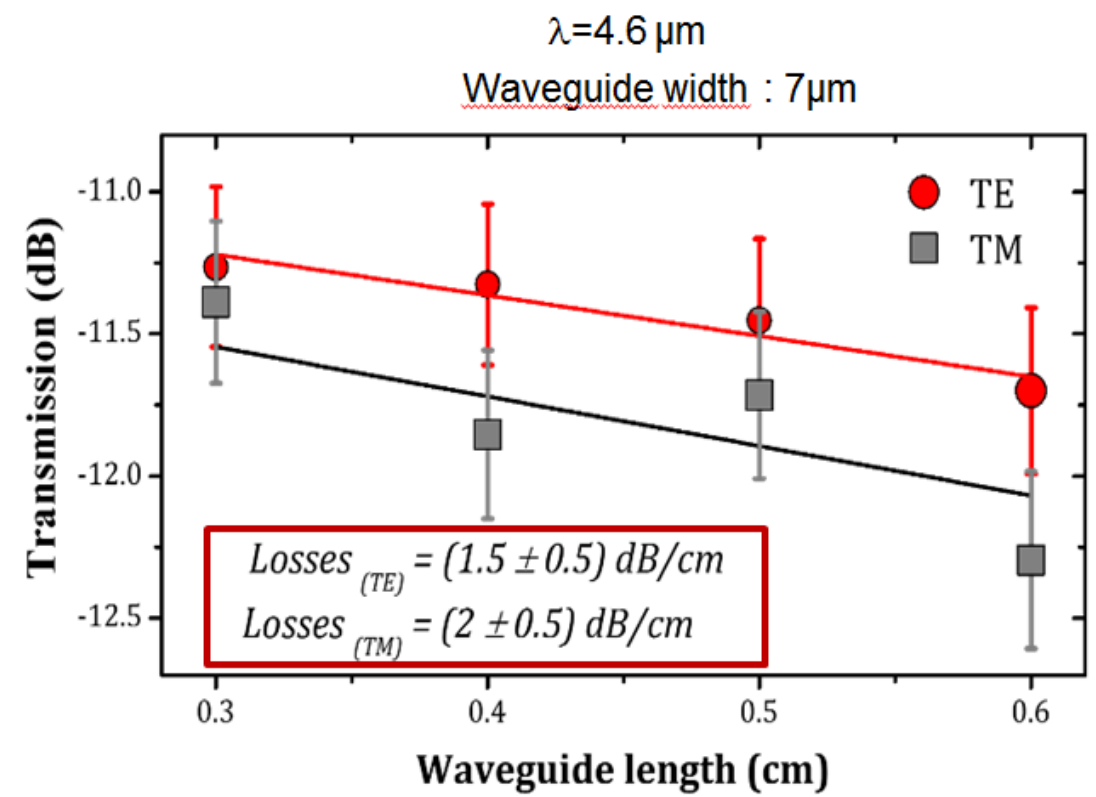

Figure 3. Characterization of the $7 \mu \mathrm{m}$-width waveguide using the cut-back technique: transmission as a function of waveguide length at the wavelength of $4.6 \mu \mathrm{m}$. Propagation losses of $1.5 \mathrm{~dB} / \mathrm{cm}(\mathrm{resp} .2 \mathrm{~dB} / \mathrm{cm})$ can be deduced for TE polarization (resp. TM polarization) 


\section{DESIGN OF GRADED SIGE WAVEGUIDES FOR OPTIMAL NON-LINEAR OPERATION}

Following the successful demonstration of good mode confinement and low optical loss in Ge-rich SiGe waveguides, the waveguide design can be optimized for non-linear operation. Indeed the strong Kerr effect of Ge and Ge-rich SiGe alloys open a new route towards the implementation of efficient mid-IR non-linear devices based on these structures [8].

The requirement to enhance non-linear effects in waveguides is to achieve simultaneously a good optical mode confinement and a flat anomalous dispersion over the considered wavelength range. Those conditions have thus been studied in Ge-rich SiGe materials.

From the waveguide reported in Fig 1, several changes have been made. First to optimize the mode confinement in a large spectral range and to optimize the overlap of the mode with Ge-rich region, it has been chosen to remove the $2 \mu \mathrm{m}$ thick constant composition layer and to use only the graded-index $\mathrm{Si}_{1-\mathrm{x}} \mathrm{Ge}_{\mathrm{x}}$ layer as the core of the waveguide, as illustrated in Fig 4. The thickness of this layer has been optimized in order to obtain a good confinement while maintaining a low threading dislocation density (TDD) due to the lattice mismatch between Si and Ge. This thickness is thus fixed to $6 \mu \mathrm{m}$. Then a rib waveguide can be defined by etching. D is the etching depth and W the waveguide thickness. Interestingly the use of a graded SiGe layer to confine the light vertically allows a unique "resizing" effect that optimize the mode size for each wavelength, as shown in fig 4.b. In addition W and D can be chosen in order to optimize the dispersion of the optical mode over the considered wavelength range. This allows us to define $\mathrm{W}=\mathrm{D}=4 \mu \mathrm{m}$ as an interesting design, combining both flat anomalous dispersion as reported in Fig 4.d as well as a tight mode confinement. Indeed very competitive values of the effective area of the optical mode lower than $10 \mu \mathrm{m}^{2}$ are obtained up to $8 \mu \mathrm{m}$ wavelength, and this value is even reduced to around $4.5 \mu \mathrm{m}^{2}$ at $3 \mu \mathrm{m}$ - wavelength, as illustrated in Fig 4.c.
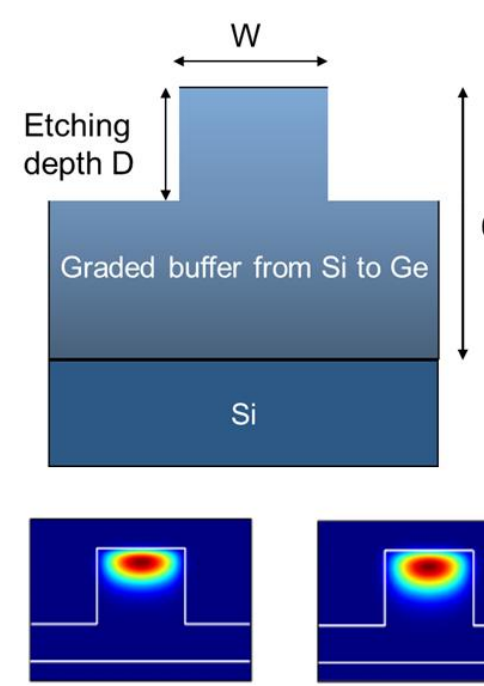

$\lambda=3 \mu m$

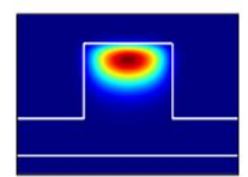

$\lambda=4 \mu m$
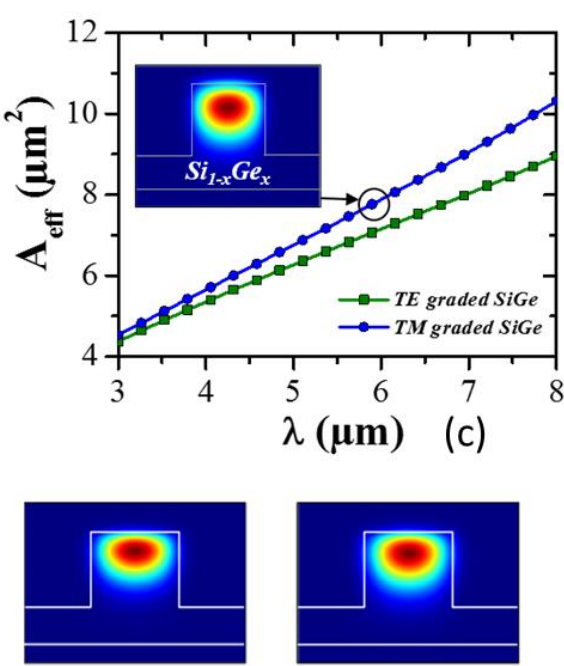

$\lambda=5 \mu m$

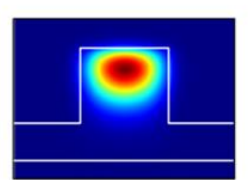

$\lambda=6 \mu m$
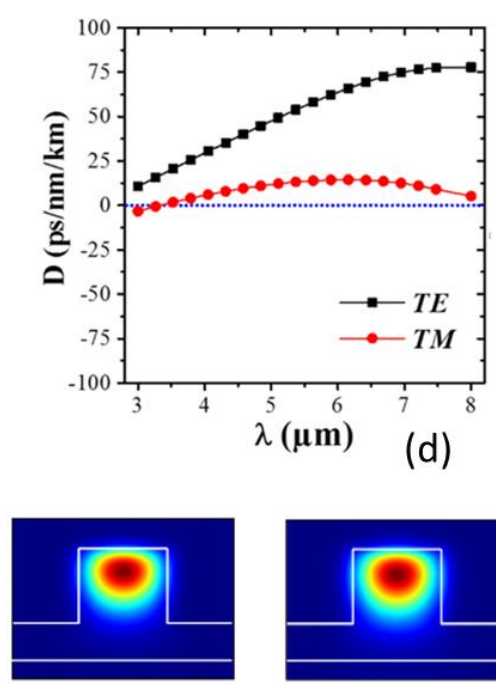

$\lambda=7 \mu m$

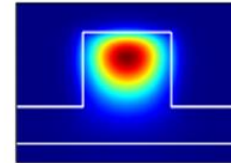

$\lambda=8 \mu m$

(b)

Figure 4. Design of Ge-rich SiGe waveguides for non-linear operation (a): cut-view of the epilayer (b) : optical mode calculation for $\mathrm{W}=\mathrm{D}=4 \mu \mathrm{m}$, and for wavelengths from 3 to $8 \mu \mathrm{m}$ (TE mode) (c) : effective area as a function of the wavelength for TE and TM optical modes. ( $\mathrm{W}=\mathrm{D}=4 \mu \mathrm{m})$

Finally the effective nonlinear parameter $\left(\gamma_{\text {eff }}\right)$ of the graded-index $\mathrm{Si}_{1-x} \mathrm{Ge}_{x}$ waveguide has been calculated and is reported in Fig 5 [10]. As expected, the graded-index $\mathrm{Si}_{1-\mathrm{x}} \mathrm{Ge}_{\mathrm{x}}$ waveguide with $\mathrm{W}=4 \mu \mathrm{m}$ and $\mathrm{D}=4 \mu \mathrm{m}$ provides a maximum 
value of $\gamma_{\text {eff }} \approx 10 \mathrm{~W}^{-1} \mathrm{~m}^{-1}$ for both polarizations at $\lambda=3 \mu \mathrm{m}$, followed by a gradual decrease for larger wavelengths down to $\gamma \sim 0.6 \mathrm{~W}^{-1} \mathrm{~m}^{-1}$ for $\lambda=8 \mu \mathrm{m}$. Such values combined with the dispersion characteristics (flat anomalous dispersion) reported in Fig 4.d are very promising for achieving supercontinuum generation in a wide spectral bandwidth [11].

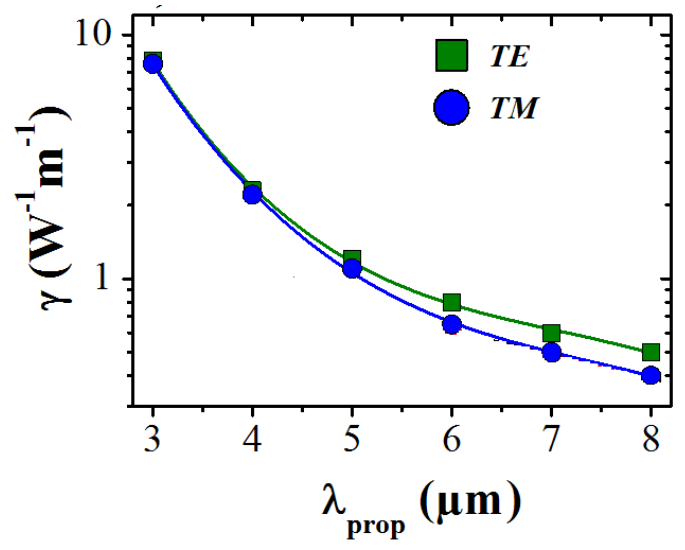

Figure 5. Calculated nonlinear parameter $\gamma_{\mathrm{eff}}$ of the quasi-TE (squares) and quasi-TM (circles) optical modes as a function of the wavelength.

\section{CONCLUSION.}

As a conclusion, Ge-rich SiGe waveguides on graded SiGe buffer layer have been studied as a fundamental building block for mid-IR integrated photonic integrated circuits. First it has been shown that these waveguides demonstrate low loss in mid-IR. $1.5 \mathrm{~dB} / \mathrm{cm}$ (resp. $2 \mathrm{~dB} / \mathrm{cm}$ ) propagation losses have been demonstrated at $4.6 \mu \mathrm{m}$ wavelength. Then a new design, based on light confinement in the graded SiGe layer has been proposed for non-linear operation, as it allows simultaneously a tight mode confinement as well as interesting dispersion properties for wideband supercontinuum generation. Interestingly, the effective nonlinear parameter has been estimated between 1 and $10 \mathrm{~W}^{-1} \mathrm{~m}^{-1}$. Such structures have thus a unique potential for being a major building block of mid-infrared photonic integrated circuits.

\section{ACKNOWLEDGMENT.}

This project has received funding from the European Research Council (ERC) under the European Union's Horizon 2020 research and innovation program (grant agreement $\mathrm{N}^{\circ} 639107-$ INsPIRE).

\section{REFERENCES}

[1] R. Soref, "Mid-infrared photonics in silicon and germanium," Nat. Photonics, 4(8), 495-497 (2010).

[2] Y-C Chang et al, "Low-loss germanium strip waveguides on silicon for the mid-infrared" Optics Letters, 37 (14), 2883 (2012).

[3] A. Malik et al, "Germanium-on-Silicon Mid-Infrared Arrayed Waveguide Grating Multiplexers", IEEE Phot. Technol. Letters, 25 (18), $1805-1808$ (2013)

[4] C. Alonso-Ramos et al, "Germanium-on-silicon mid-infrared grating couplers with low-reflectivity inverse taper excitation", Optics Letters 41 (18),4324 (2016)

[5] M. Nedeljkovic et al "Surface-Grating-Coupled Low-Loss Ge-on-Si Rib Waveguides and Multimode Interferometers", IEEE Phot. Technol. Letters, 27, 1040-1043 (2015) 
[6] M. Brun, et al "Low loss SiGe graded index waveguides for mid-IR applications," Optics express, 22(1), 508518 (2014).

[7] P. Chaisakul, et al, "Integrated germanium optical interconnects on silicon substrates", Nature Photonics 8, 482-488 (2014)

[8] N. K. Hon et al, "The third-order nonlinear optical coefficients of $\mathrm{Si}, \mathrm{Ge}$, and $\mathrm{Si}_{1-\mathrm{x}} \mathrm{Ge}_{\mathrm{x}}$ in the midwave and longwave infrared, Journal of Applied Physics, 110, 011301 (2011).

[9] S. Marchionna, A. Virtuani, M. Acciarri, G. Isella, and H. von Kaenel, "Electron-beam-induced current imaging for the characterisation of structural defects in $\mathrm{Si}_{1-\mathrm{x}} \mathrm{Ge}_{\mathrm{x}}$ films grown by LE-PECVD” Mat. Sci. Semicond. Process. 9 (4-5) 802 (2006).

[10] J-M. Ramirez et al, "Ge-rich graded-index $\mathrm{Si}_{1-\mathrm{x}} \mathrm{Ge}_{\mathrm{x}}$ waveguides with broadband tight mode confinement and flat anomalous dispersion for nonlinear mid-infrared photonics" submitted to Optics Express (2017).

[11] L. Carletti, M. Sinobad, P. Ma, Y. Yu, D. Allioux, R. Orobtchouk, M. Brun, S. Ortiz, P. Labeye, J. M. Hartmann, S. Nicoletti, S. Madden, B. Luther-Davies, D. J. Moss, C. Monat, and C. Grillet, "Mid-infrared nonlinear optical response of Si-Ge waveguides with ultra-short optical pulses," Optics express, 23(25), 3220232214 (2015). 